\title{
ERROR ANALYSIS OF THE DISPERSION-COMPENSATED WIDE-BAND POLARIZATION SAGNAC INTERFEROMETER
}

\author{
Jingping Zhu ${ }^{1,2}$, , Kang Zong ${ }^{1}$, François Goudail ${ }^{2}$, Ning Zhang ${ }^{1}$, Xun Hou ${ }^{1}$ \\ ${ }^{1}$ Key Laboratory for Physical Electronics and Devices of the Ministry of Education \& Shaanxi Key Lab of Information Photonic \\ Technique, Xi'an Jiaotong University, 710049 Xi'an, China - jpzhu@xjtu.edu.cn \\ ${ }^{2}$ Laboratoire Charles Fabry, Institut d'Optique, CNRS, 91127 Palaiseau, France
}

KEY WORDS: Sagnac Interferometer, Channeled Polarimetry, Reconstruction Errors, Blazed Gratings, Polarization Beam Splitter

\begin{abstract}
:
Imaging polarimetry can obtain two-dimensional intensity distribution information and its corresponding states of polarization from the target simultaneously. Sagnac polarization imaging interferometer is one of the typical channeled polarimetry technologies. By splitting the incident light through the triangular optical structure, the polarization information of the target is modulated into the interference fringes and can be obtained by the demodulation algorithm. The non-ideal optical elements in the system will cause reconstruction errors. This article analyses several factors that affect the measurement accuracy, including the splitting ratio of polarization beam splitter and the diffraction efficiency of the blazed grating. We derive the modified intensity formula and obtain interference patterns through numerical simulations. By calculating the modulation degree of interference fringes and reconstruction error under different non-ideal parameters, we have come to the conclusion that to meet the requirement of modulation degree $>0.5$ and reconstruction error $<10 \%$, the beam splitting ratio of the PBS should be $\alpha<1.22$. The diffraction efficiency ratio of spolarization to p-polarization of the blazed grating should be $\eta_{s} / \eta_{p}>0.83$, and the first-order diffraction efficiency should be $\eta_{1}>79 \%$.
\end{abstract}

\section{INTRODUCTION}

Imaging polarimetry is developed to measure the two dimensional spatial distribution of intensity and strokes parameters of the target simultaneously (Tyo, 2006). Compared with the traditional intensity detection, the polarimetric information has the ability to enhance the contrast between the target and the background, improve the detection and recognition capabilities and accuracy. It is now becoming a valuable tool in the field of remoting sensing (Miller and Dereniak, 2012), biomedicine (Luo and Zhang, 2014), materials (Li et al., 2015) and so on.

Conventional methods use rotating elements to perform multiple measurements to obtain the Stokes parameters. Mechanically rotating components can complicate the structure of the system and make it impossible to measure the polarization information of non-static targets in real time (Giudicotti and Brombin, 2007). Later, simultaneous detection methods such as division of amplitude (DoAP) (Tu et al., 2017), division of aperture (DoA) (Pezzaniti and Chenault, 2005), and division of FPA (DoF) (Gruev et al., 2010) were developed, which can acquire the Stokes parameters of the target in a snapshot. However, these methods usually require the use of polarization analysing optics, such as rotation polarizer, micro waveplate, lens array or micro polarizer array, resulting in complex system structure, difficult alignment, and registration error.

The channeled polarimetry technique was proposed by K. Oka (Oka, 2003), in which Stokes vectors can be modulated onto spatial interference fringes. Kudenov (Kudenov et al., 2009) developed a dispersion compensated polarization Sagnac interferometer, which could acquire linear polarization state of the target in white light based on two polarization gratings. But the reconstruction errors of the system are relatively high due to the non-ideal optical elements, including the splitting ratio of polarization beam splitter (PBS) and the diffraction efficiency of the blazed gratings.

In this paper, we present a modification of Kudenov's system, and three main factors that affect the reconstruction accuracy are analysed.

\section{PRINCIPLE OF THE DISPERSION-COMPENSATED WIDE-BAND POLARIZATION SAGNAC INTERFEROMETER (DCWPSI)}

Compared with the Kudenov's original structure, we move the two blazed diffraction gratings from the right-angle side to the bevelled side. The distance between the two gratings can be better adjusted on the hypotenuse, therefore the system can be miniaturized. The optical layout of the modified DCWPSI is depicted in Figure 1.

Incident light initially reflected by the wire-grid beam splitter (WGBS) is reflected by mirror M1 and then diffracted by grating G1 into the +1 order. These dispersed rays propagate to grating G2, where they are diffracted into the zero order, thus removing the diffraction angle imposed by G1. The rays exit the system parallel to the optical axis with an offset. Conversely, the beam transmitted by the WGBS is reflected by M2 and diffracted by $\mathrm{G} 2$ into its +1 order, diffracted by G1 into the zero order, and exits the system with the same offset. Assuming small angles and G1, G2 are identical, the shear $S_{D C W P S I}$ is

$$
S_{D C W P S I}=2 \frac{m \lambda L}{d}
$$

\footnotetext{
* Corresponding author
} 


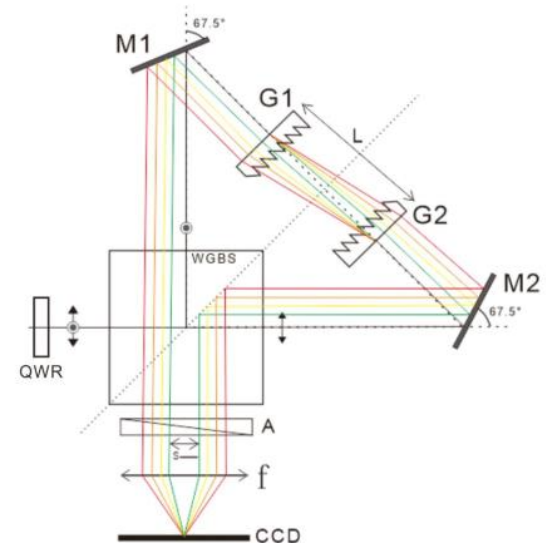

Figure 1. Schematic setup of the modified DCWPSI

Where $\mathrm{m}$ is the order of diffraction, $\lambda$ is the wavelength of the incident light, $\mathrm{L}$ represents the distance between $\mathrm{G} 1$ and $\mathrm{G} 2$, and $\mathrm{d}$ is the period of the grating.

Once the two beams go through the polarizer and are combined by the objective lens, they produce interference fringes on the CCD Camera. A quarter-wave retarder (QWR) oriented at $45^{\circ}$ in front of the system is used to measure linear polarization $\left(\mathrm{S}_{0}\right.$, $\mathrm{S}_{1}$, and $\mathrm{S}_{2}$ ). The intensity on the FPA is:

$$
\begin{aligned}
& I(x, y)=\frac{1}{2} S_{0}+\frac{1}{2} S_{2} \cos \left(2 \pi U_{D C W P S I} x_{i}\right) \\
& -\frac{1}{2} S_{1} \sin \left(2 \pi U_{D C W P S I} x_{i}\right)
\end{aligned}
$$

The carrier frequency is:

$$
U_{D C W P S I}=\frac{2 m L}{d f}
$$

Where $f$ is the focal length of the objective lens. The dispersion caused by the wavelength is removed. The Stokes parameters $S_{1}$ and $\mathrm{S}_{2}$ are amplitude modulated onto different carrier frequencies, while $\mathrm{S}_{0}$ remains un-modulated.

The Fourier transform of the Eq. (2) is:

$$
\begin{aligned}
& F(\tilde{I})=\frac{1}{2} A_{0}\left(f_{x}, f_{y}\right)+\frac{1}{4} A_{21}\left(f_{x}-U, f_{y}\right) \\
& +\frac{1}{4} A_{21}^{*}\left(-f_{x}+U,-f_{y}\right)
\end{aligned}
$$

Where $\left(f_{x}, f_{y}\right)$ is the spatial frequency. It can be seen from Eq. (4) that the frequency domain spectrum corresponding to $\tilde{I}\left(f_{x}, f_{y}\right)$ contains 3 parts, concentrated at $h=(0,0)$ and $( \pm U, 0)$ respectively. Extract the interested regions by choosing the appropriate frequency filter, and then use the inverse Fourier transform to obtain different Stokes parameters as:

$$
\begin{gathered}
S_{0}=f^{-1}\left(A_{0}(x, y)\right) \\
S_{2}=\operatorname{real}\left[f^{-1}\left(A_{21}\left(f_{21}-U, f_{y}\right)\right) e^{-i U x}\right] \\
S_{1}=\operatorname{imag}\left[f^{-1}\left(A_{21}\left(f_{21}-U, f_{y}\right)\right) e^{-i U x}\right]
\end{gathered}
$$

The liner polarization information of the target can be solved from Eq. (5).

\section{ERROR ANALYSIS OF THE DCWPSI}

When two beams $\mathrm{I}_{1}$ and $\mathrm{I}_{2}$ with the same vibration direction and frequency meet in space, the interference intensity is:

$$
I=I_{1}+I_{2}+\sqrt{I_{1} I_{2}} \cos \left(\varphi_{2}-\varphi_{1}\right)
$$

Where $\phi_{1}$ and $\phi_{2}$ are the initial phase of the two beams respectively.

The modulation degree of interference fringes is defined as:

$$
V=\frac{I_{\max }-I_{\min }}{I_{\max }+I_{\min }}=\frac{2 \sqrt{I_{1} I_{2}}}{I_{1}+I_{2}}
$$

It can be seen from Eq. (7) that when $I_{1}$ and $I_{2}$ are equal, the modulation degree of interference fringes is the highest. Due to the limitation of the actual processing technology, the splitting ratio of the polarizing beam splitter is not ideal 50:50. After the incident light is diffracted by the blazed grating, other orders of stray light will enter the system in addition to the first order, resulting in contrast reduction of the interference fringes.

Next, we will discuss three main factors that affect the reconstruction accuracy, including the splitting ratio of polarization beam splitter, the diffraction efficiency ratio of spolarization to p-polarization of the blazed grating and the diffraction efficiency of other orders.

\subsection{Beam splitting ratio of the polarizing beam splitter}

The PBS transmits p-polarized light and reflects s-polarized light. Let the ratio of the p-polarized component and the spolarized component be $\alpha$. When the incident light $\left[\begin{array}{ll}E_{x} & E_{y}\end{array}\right]^{T}$ passes through the PBS, the reflected s-polarized and transmitted p-polarized components are $\alpha E_{x}$ and $E_{y}$, respectively. By the ray tracing calculation, we can obtain the interference fringe intensity on the CCD is:

$$
\begin{aligned}
& I^{\prime}=\frac{1}{4}(\alpha+1) S_{0}+\frac{1}{2} \sqrt{\alpha} S_{2} \cos \left(2 \pi U x_{i}\right) \\
& -\frac{1}{2} \sqrt{\alpha} S_{1} \sin \left(2 \pi U x_{i}\right)-\frac{1}{4}(\alpha-1) S_{3}
\end{aligned}
$$

Compared with Eq. (2), the extra $\mathrm{S}_{3}$ term in Eq. (8) will cause reconstruction error in $\mathrm{S}_{0}$ and affect the demodulation accuracy of $S_{1}$ and $S_{2}$.

Set the Stokes parameter of the incident light as $S=\left[\begin{array}{llll}1 & 0.7 & 0.71 & 0.1\end{array}\right]^{T}$, and the interference patterns simulated under different beam splitting ratios are shown in Figure 2. (Picture on the right in the red dotted line is a partial enlargement of the interference fringes.) 


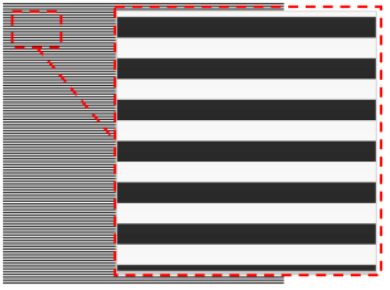

(a)

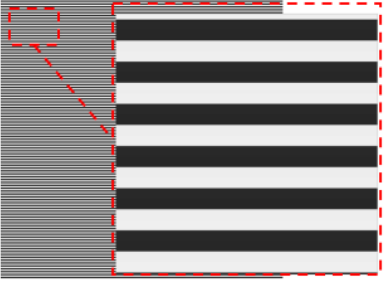

(c)

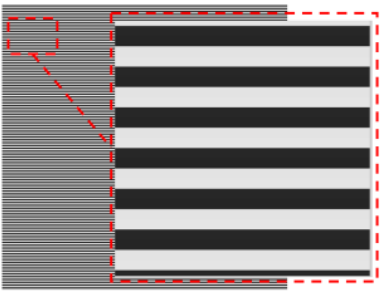

(b)

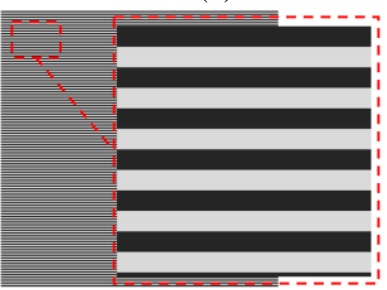

(d)
Figure 2. (a) (b) (c) (d) are the interference patterns when $\alpha$ is $1,1.1,1.2$ and 1.3 respectively

Take Figure 2 (d) as an example, the intensity at $\mathrm{x}=200$ is shown in Figure 3.

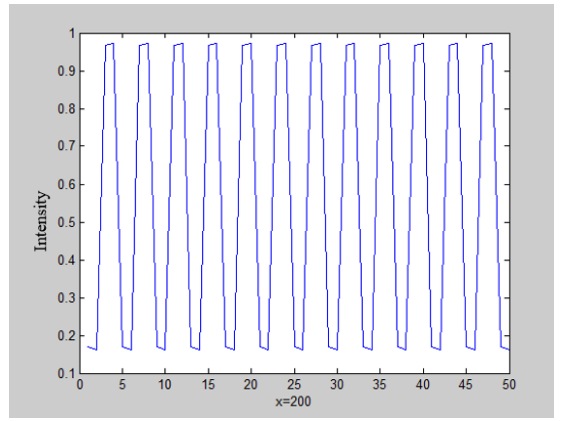

Figure 3. The intensity of interference fringes at $x=200$

The modulation degree of Figure 3 is calculated as 0.71 through eq. (7).

The spectrogram of the interferogram Figure 2. (d) is shown in Figure.4(a) by doing the Fourier transform. Figure.4 (b) is 3-D perspective of spectrogram.

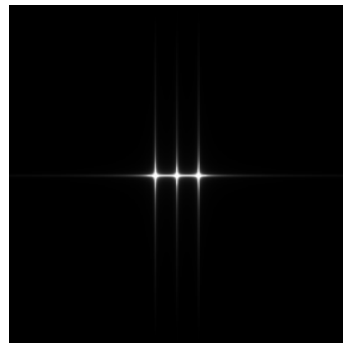

(a)

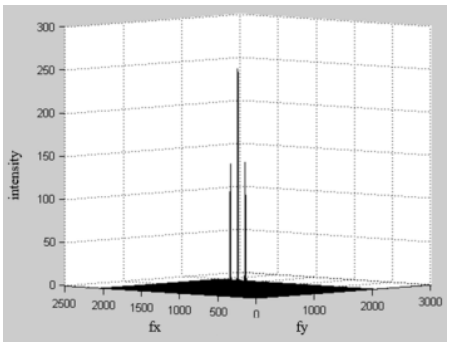

(b)
Figure 4. Fourier spectrogram. (a)Top view. (b)3-D view.

Select a suitable rectangular filter as shown in Figure 5, do inverse Fourier transform, and calculate the degree of the linear polarization (DoLP) as 0.86. Compared with the DoLP of the original incident light, the reconstruction error is $13.5 \%$.

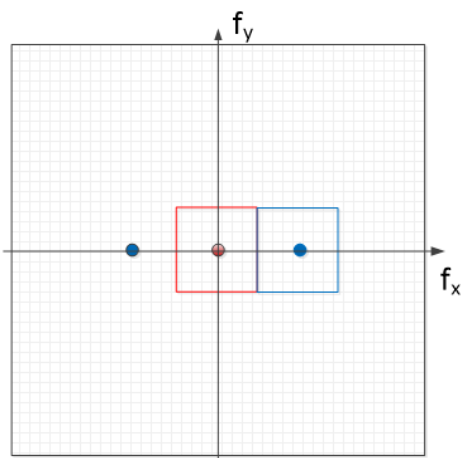

Figure 5. The red and blue marked squares denote the data area that carries the information of $S_{0}$ and $S_{21}$

In the same way, we can calculate the modulation degree of Figure 2 (a) - (c) is all 0.71, which satisfies the requirement of modulation degree $>0.5$. But The reconstruction errors are $0 \%$, $4.5 \%$, and $9.0 \%$, respectively. To meet the error requirement (e $<10 \%$ ), the beam splitting ratio should be $\alpha<1.22$.

\subsection{The diffraction efficiency ratio of s-polarization to $p$ - polarization of the blazed grating}

The difference in the p-polarized and s-polarized diffraction efficiency of the blazed grating will affect the intensity of the two light beams emitted by the DCWPSI. Let the p-polarized and s-polarized diffraction efficiency of the blazed grating be $\eta_{p}$ and $\eta_{s}$ respectively, and the calculated intensity of the interference fringes on the $\mathrm{CCD}$ is:

$$
\begin{aligned}
& I^{\prime \prime}=\frac{1}{4}\left(\eta_{p}^{2}+\eta_{s}^{2}\right) S_{0} \\
& +\frac{1}{2} \eta_{p} \eta_{s}\left(S_{2} \cos \left(2 \pi U x_{i}\right)-S_{1} \sin \left(2 \pi U x_{i}\right)\right)-\frac{1}{4}\left(\eta_{p}^{2}-\eta_{s}^{2}\right) S_{3}
\end{aligned}
$$

Similar to the situation in Section 3.1, the extra $S_{3}$ term in Eq. (9) will cause errors in the reconstruction of $S_{0}, S_{1}$ and $S_{2}$.

Set the Stokes parameter of the incident light as $S=\left[\begin{array}{llll}1 & 0.7 & 0.71 & 0.1\end{array}\right]^{T}$ and $\eta_{p}=1$. The simulated interference patterns are shown in Figure 3 when $\eta_{s}$ is 1, 0.9, 0.8 , and 0.7 , respectively.

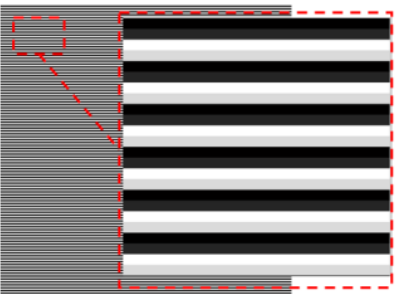

(a)

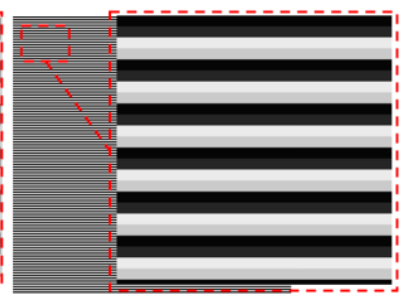

(b) 


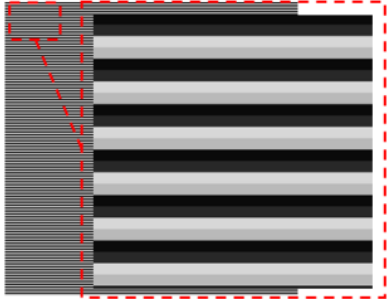

(c)

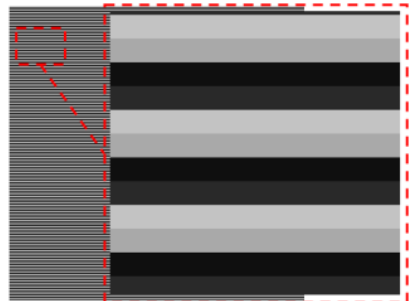

(d)
Figure 6. (a) (b) (c) (d) are the interference patterns when $\eta_{p}=1$ and $\eta_{s}$ is $1,0.9,0.8,0.7$ respectively.

It can be seen from Figure 6 that as the ratio between $\eta_{p}$ and $\eta_{s}$ becomes larger, the contrast of interference fringes will drop sharply. Use the same method as in Section 3.1, we can calculate the modulation degree of the four graphs in Figure 3 is $1,0.96,0.91,0.85$, and the reconstruction error is $0 \%, 6.0 \%$,

$11.8 \%, 17.6 \%$ respectively. To meet the demodulation requirement of modulation degree $>0.5$ and error requirement of e $<10 \%$, the diffraction efficiency ratio of the p-polarization to the s-polarization of the blazed grating should be $\eta_{s} / \eta_{p}>0.83$.

\subsection{Diffraction efficiency of other orders of blazed grating}

Ideally the blazed grating will diffract most of the energy up to the first order. However, due to imperfect manufacturing process, other orders diffracted by the grating will also enter the system, causing interference fringes aliasing and contrast reduction, similar to the dispersion caused by the wavelength in the traditional Sagnac interferometer.

Carrier frequency in the DCWPSI is proportional to the diffraction order $\mathrm{m}$. Set the diffraction efficiency of other orders as $\eta_{m}$, the calculated intensity of the interference fringes on the $\mathrm{CCD}$ is:

$$
\begin{aligned}
& I^{\prime \prime}=\frac{1}{2} S_{0}+ \\
& \sum_{\mathrm{m}=1}^{d / \lambda_{\min }}\left(\left(\frac{1}{2} \eta_{m}^{2} S_{2} \cos \left(2 \pi U x_{i}\right)-\frac{1}{2} \eta_{m}^{2} S_{1} \sin \left(2 \pi U x_{i}\right)\right)\right)+\eta_{0}^{2} S_{0}
\end{aligned}
$$

It can be seen from Eq.(10) that the middle term of $I$ "' is the superposition of diffractive fringes of different frequencies, which will cause the aliasing of fringes. The last term is the 0order light without diffraction, resulting in reduction of the fringe contrast.

Set the Stokes parameter of the incident light as $S=\left[\begin{array}{llll}1 & 0.7 & 0.71 & 0.1\end{array}\right]^{T}$ and the other diffraction order of blazed grating up to three. Use the matrix $\eta=\left[\begin{array}{llll}\eta_{0} & \eta_{1} & \eta_{2} & \eta_{3}\end{array}\right]$ to express the diffraction efficiency of the $0,1,2$ and 3 order. The simulated interference patterns are shown in Figure 7 when the diffraction efficiency values of different orders

$\eta_{a}=\left[\begin{array}{llll}0 & 1 & 0 & 0\end{array}\right] \quad, \quad \eta_{b}=\left[\begin{array}{llll}0.1 & 0.8 & 0.08 & 0.02\end{array}\right]$

$$
\begin{aligned}
& \eta_{c}=\left[\begin{array}{llll}
0.15 & 0.7 & 0.1 & 0.05
\end{array}\right] \\
& \eta_{d}=\left[\begin{array}{llll}
0.2 & 0.6 & 0.1 & 0.1
\end{array}\right], \text { respectively. }
\end{aligned}
$$

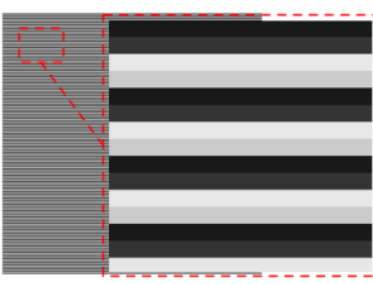

(a)

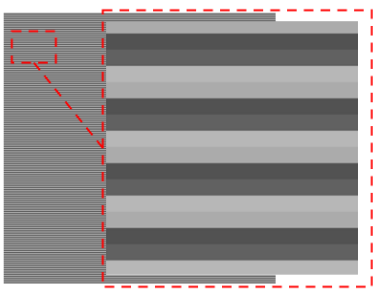

(c)

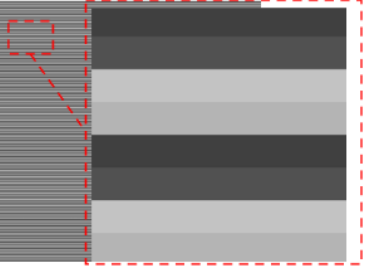

(b)

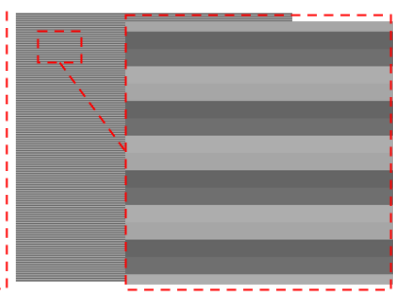

(d)
Figure 7. (a) (b) (c) (d) are the interference patterns when $\eta$ takes four different sets of values.

We calculate the modulation degree of the interference fringes in the four cases is $0.81,0.51,0.38$ and 0.27 , and the reconstruction error is $0 \%, 2.0 \%, 4.5 \%$, and $8.0 \%$, respectively. To meet the requirements of modulation degree $>0.5$ and $\mathrm{e}<10 \%$, diffraction efficiency of the first order should be $\eta_{1}>79 \%$.

\section{CONCLUSION}

In conclusion, we discussed three main influencing factors which will cause reconstruction errors of DCWPSI, including the beam-splitting ratio of the polarizing beam splitter, the diffraction efficiency ratio of p-polarization to s-polarization of the blazed grating and the diffraction efficiency of the other orders. By setting the Stokes vector of the incident light and changing the influence factors, it is calculated that to meet the requirement of demodulation and error (modulation degree $>0.5$, $\mathrm{e}<10 \%$ ), the beam splitting ratio of the PBS should be $\alpha<1.22$. The diffraction efficiency ratio of s-polarization to $\mathrm{p}$ polarization should be $\eta_{s} / \eta_{p}>0.83$, and the first-order diffraction efficiency should be $\eta_{1}>79 \%$. The calculation results of this paper provide guidance for the device selection of the channeled polarimeter based on Sagnac polarization interferometer.

\section{REFERENCES}

Giudicotti, L., Brombin, M., 2007. Data analysis for a rotating quarter-wave, far-infrared Stokes polarimeter. Applied Optics, $46,2638-2648$.

Gruev, V., Perkins, R., York, T., 2010. CCD polarization imaging sensor with aluminum nanowire optical filters. Optics Express, 18, 19087-19094. 
Kudenov, M.W., Jungwirth, M.E.L., Dereniak, E.L., Gerhart, G.R., 2009. White light Sagnac interferometer for snapshot linear polarimetric imaging. Optics Express, 17, 22520-22534.

Li, Y.F., Zhang, J.Q., Qu, S.B., Wang, J.F., Zheng, L., Zhou, H., $\mathrm{Xu}, \mathrm{Z}$., Zhang, A.X., 2015. Wide-band circular polarizationkeeping reflection mediated by metasurface. Chinese Physics $B$, 24.

Luo, G., Zhang, M., 2014. Backscattering from small-scale breaking wave turbulence structure generated by FLUENT. Chinese Physics B, 23.

Miller, D.A., Dereniak, E.L., 2012. Selective polarization imager for contrast enhancements in remote scattering media. Applied Optics, 51, 4092-4102.

Oka, K., 2003. Compact complete imaging polarimeter using birefringent wedge prisms.

Pezzaniti, J.L., Chenault, D.B., 2005. A division of aperture MWIR imaging polarimeter. Proceedings of Spie the International Society for Optical Engineering, 44, 515-533.

Tu, X.Z., Spires, O.J., Tian, X.B., Brock, N., Liang, R.G., Pau, S., 2017. Division of amplitude RGB full-Stokes camera using micro-polarizer arrays. Optics Express, 25, 33160-33175.

Tyo, J.S., 2006. Review of passive imaging polarimetry for remote sensing applications. APPLIED OPTICS. 